\title{
1979 joint DEC and microprocessor users group report
}

\author{
DORIS AARONSON and MISHA PAVEL \\ New York University, New York, New York 10003
}

\begin{abstract}
A summary is provided of the format and results of a meeting involving users of microprocessors and of Digital Equipment Corporation computers.
\end{abstract}

The DEC and microprocessor technical sessions were run sequentially in the same conference room in order to effectively continue the user groups. This action was taken for three reasons. First, a membership survey indicated almost a $50 \%$ overlap of On-Line members interested in these two groups. Second, microprocessors are now visibly present in many DEC labs. DEC's line of LSI-11 microprocessors has become popular among psychologists needing only a small stand-alone machine. And finally, many psychologists with larger systems are adding DEC and non-DEC microprocessors to their DEC minis as display controllers or as entire remote subject stations in network configurations.

The formal part of the presentation in the DEC session consisted of three papers describing particular systems based on the DEC LSI-11 microprocessor series. These papers, which can be found under Session $X$ in this BRMI issue, were given by W. L. Palya and A. F. Doyle, by A. F. Doyle and W. L. Palya, and by W. O. Shaffer and K. W. Scholz.

The microprocessor session experimented with a new and well-received poster format. Three users set up microprocessor demonstrations with poster-type explanations, while they were available for discussions with individual On-Line members. In addition, they gave brief summary presentations to the group as a whole. L. E. Wood, Brigham Young University, demonstrated the Apple II system under the title "An Intelligent Program to Teach Logical Thinking." T. B. Perera, Montclair State College, presented "Twenty Experimental Psychology Laboratory Sessions Using the TRS-80." Also, J. D. Hollan, University of California at San Diego, used the Terak LSI-11-based system to demonstrate experimental programs based on the UCSD PASCAL software.

The discussion following the formal part of the session was concentrated on two main topics: the multiprocessor configurations for real-time experiments and the problems arising from combining DEC and non-DEC hardware.

The first question concerned the type of systems with one host computer connected to a number of satellites dedicated to real-time tasks. The discussion focused on the advantages and disadvantages of various configurations and the conditions under which the multiproces- sor system is found to be cost effective. The critical decision factors brought up in the discussion included the following: (1) availability of technical support and maintenance funds, (2) data bandwidth requirements for specific experimental situations, (3) scheduling and amount of usage of the system, (4) user sophistication, (5) cost of ongoing operations, and (6) capability for updating and expanding.

The main advantages of the distributed system include the potentially low cost of satellite stations, along with the computing power of a larger machine having more sophisticated peripherals. The satellite user can benefit from having powerful software supported by the host for program development and data analysis. Regarding the software, the distributed system can be especially convenient. Because the real-time task is carried out by the satellite, the host's timesharing operating system doesn't need real-time capabilities. For example, the operating system UNIX developed by Bell Laboratories which doesn't ordinarily support real-time tasks can be used in a psychological laboratory. Thus, this is a feasible alternative to DEC's timesharing operating systems, which are relatively inflexible.

On the other hand, stand-alone microprocessor systems that fulfill many of our psychology applications can now be purchased for substantially less than minicomputers. This price advantage becomes especially important when one considers that peripherals are cheaper to buy and to interface for micros than for minis. The dedicated system approach is particularly attractive for users with large data throughput requirements such as research in vision, speech, and physiology.

Even after a decision has been made in favor of a distributed system, there are a number of important characteristics that must be determined by the system designer. One of the most important parameters is the tightness of coupling, determining the degree of independence of each machine. For example, very tightly coupled systems may be more efficient but require more than one processor operating simultaneously-a potential source of reliability problem. Another factor concerns the interprocessor communication hardware. Although some users have connected microprocessors to their host minis via high-speed parallel channels, most users are employing a slower 
serial RS-232 interface. The main reason for this type of connection is simplicity: Anything that can mimic a terminal can be supported with little or no change in the host's systems.

A common practice in many laboratories is to configure the system by combining equipment from a number of different vendors. In particular, several users now have running systems with DEC minicomputer hosts and non-DEC satellites. Others have non-DEC interfaces or peripherals. Consequently, users are having the usual installation and service problems that go along with mixing any DEC and non-DEC equipment. When there are initial problems of interfacing, to make the DEC and the non-DEC hardware compatible, neither company wants to get involved with the other's product. After the system has been running for a while and components begin to fail, neither company wants to (or knows how to) troubleshoot the other's equipment.

In response to these $\mathrm{DEC} /$ non-DEC problems, experienced users of mixed systems had several suggestions. (1) Users considering a mixed DEC/non-DEC system are best advised to have their own experienced in-house engineering staff, even in addition to maintaining service contracts. (2) When intersystem problems arise, users found the non-DEC vendors both more knowledgeable and more willing to help than DEC service people. (3) When initially developing a mixed system, find a friend with a similar working system and copy it. (4) When problems develop later, call a friend; he or she may have already dealt with that or related problems.

At the end of the meeting, the idea of developing a list of computer friends experienced with mixed systems was further pursued. During the year we will try to develop such a roster for distribution at the next On-Line meeting. Persons who are willing to be on such a list of "computer friends" should send to D. Aaronson (NYU Psychology, 6 Washington Place, Room 858, New York, New York 10003) their name, address, phone number and a list of their computer hardware and operating system(s). We already have a few computer friends as a result of this postmeeting discussion. Adam Reed (Psychology Department, New School for Social Research, 65 Fifth Avenue, New York, New York 10003) is familiar with mixing DEC and non-DEC (particularly Apple) computers. William Palya (Psychology Department, Jacksonville State University, Jacksonville, Alabama 36235) is quite experienced in the problems of mixing DEC and non-DEC equipment in general. We recommend his article on LSI-11-compatible products (Palya, 1978). Allan Doyle, currently at Massachusetts Institute of Technology and soon to be with the DEC national office at Maynard, Massachusetts, is quite experienced with the LSI-11 series of DEC microcomputers. Finally, Steve Mullen, the DEC representative for Life Science Applications (200 Forrest Street, Marlborough, Massachusetts 01752, Mail Stop MR2-3/ M84, Phone: (617) 481-9511, extension Marlboro 6943) has been helpful to users for the past several years and is a specialist in DEC microprocessors.

\section{REFERENCE}

PALYA, W. A source handbook for Digital Equipment Corporation LSI-11-compatible products. Behavior Research Methods \& Instrumentation, 1978, 10, 488-509. 